\title{
AN INTERDISCIPLINARY APPROACH TO THE MANAGEMENT OF CRITICALLY ILL PATIENTS DURING COVID-19 PANDEMIC; AN EXPERIENCE OF A UNIVERSITY HOSPITAL IN ENGLAND
}

DOI: 10.36740/WLek202007147

\author{
Tomasz Torliński' ${ }^{1}$, Lucas Rakasz ${ }^{2}$, Barbara Wysota ${ }^{3}$, Marcin Czyż², Catherine Snelson' ${ }^{1}$ \\ 'DEPARTMENT OF ANAESTHETICS AND CRITICAL CARE, QUEEN ELIZABETH HOSPITAL BIRMINGHAM, UNIVERSITY HOSPITALS BIRMINGHAM NHS FT, \\ BIRMINGHAM, UNITED KINGDOM \\ 2DEPARTMENT OF NEUROSURGERY, QUEEN ELIZABETH HOSPITAL BIRMINGHAM, UNIVERSITY HOSPITALS BIRMINGHAM NHS FT, BIRIMINGHAM, UNITED KINGDOM \\ ${ }^{3}$ DEPARTMENT OF NEUROLOGY, QUEEN ELIZABETH HOSPITAL BIRMINGHAM, UNIVERSITY HOSPITALS BIRMINGHAM NHS FT, BIRMINGHAM, UNITED KINGDOM
}

\begin{abstract}
COVID-19 pandemic presents significant challenges in delivering safe and efficient patient care, especially during the surges. In all health care systems, provision of available critical care facilities is a scarce resource, even in normal times. Problematic is not just the limitation of physical spaces in intensive care units, but also the availability of trained personnel. The critical care model, developed in Queen Elizabeth Hospital Birmingham to cope with the surge of COVID-19 patients, is based on early implementation of an interdisciplinary approach and extensive cooperation between the branches of practice, allowing to address both challenges. The main pillars are early upskilling of non-critical care staff, creation of safe, streamlined clinical pathways, adjustment of the physical layout of critical care units and comprehensive cross-town cooperation allowing to accommodate an increased number of patients, requiring intensive care. The model was well tested in clinical practice, enabling the hospital to increase the critical care footprint by more than 200\% during the pandemic's surge between March and May 2020.
\end{abstract}

KEY WORDS: Critical care, clinical governance, interdisciplinary intensive therapy, COVID-19, medical education, pandemic

Wiad Lek. 2020;73(7):1576-1579

\section{INTRODUCTION}

COVID -19 disease initially originated in Wuhan, Hubei Province in the People Republic in China, and very quickly spread across the world, reaching the United Kingdom on the 31st of January 2020. On the 11th of March, the World Health Organization (WHO) declared COVID-19 as a worldwide pandemic. SARS$\mathrm{CoV}-2$ virus has been identified as a cause of the disease. Even before that announcement, on the $3^{\text {rd }}$ of March Department of Health and Social Care of British Government, published Coronavirus (COVID-19) action plan outlining what the health and social care system across the UK had done to tackle the coronavirus (COVID-19) outbreak and the plans for the next steps [1].

National Health Service (NHS) England, the overarching regulatory body, instructed all Hospitals to prepare for the impending surge of patients [2]. The initial set of guidelines was issued on the 17th of March 2020, providing a framework allowing for the rapid expansion of critical care services using a multidisciplinary approach [3]. The national priorities for England were designed to increase Critical Care capacity at least threefold, allowing for the provision of safe and efficient care not only for Covid-19 positive patients but also allowing for a continuous inflow of non-COVID emergencies and from health and safety point of view a provision of the safe working environment for medical staff [4].

The provision of critical care beds is a scarce resource across all countries with the United Kingdom and Poland having a very similar number of intensive care beds per 100000 inhabitants -6.6 and 6.9 respectively [5]. Our healthcare organisation - University Hospitals Birmingham NHS Foundation Trust consists of four hospitals providing secondary and tertiary care for the West Midlands region, the second most populated metropolitan area in England, with very high population density and considerable areas of social deprivation. Jointly the total provision of critical care beds across all four sites is around 110, with more than 70 beds alone available at the biggest site - Queen Elizabeth Hospital Birmingham [5].

\section{THE AIM}

The national and local expectations were that significant reconfiguration of health care provision, especially critical care, need to be made to face the challenge of COVID-19 surge. Therefore, the aim of the paper is to share our experience of organizational challenges and adjustments required in the midst of pandemic.

\section{RESPONSE TO CHALLENGES}

Based on the guidelines, published jointly by the specialist learned societies and colleges, the multimodal approach was used to prepare for the inevitable surge of the patients [6]. From the onset, it became apparent that if the surge of the patients required a two or threefold increase of critical 
care capacity, it would be impossible to continue with business as usual. An innovative way of working was needed to be established. It had also become clear that to ensure continuity of care and improve the resilience of medical staff, 12 hours resident shift ought to be introduced in place of the standard on-call arrangements. The predicted, massive surge of patients, on the one hand, precluded the division of hospitals into hot and clean sites. On the other hand, as in Poland, the model required to establish ways to continue the provision of urgent and emergency secondary and tertiary care at all sites.

The special emphasis was put on the following pathways to achieve organisational aims:

- creation of control and command centres, with regular meetings and clear daily communication at Trust, hospital and departmental levels, utilising modern media channels;

- development of critical care workforce by quick upskilling medical professionals with previous exposure and experience of the intensive care medicine to allow redeployment of staff to more occupied areas;

- increase in critical care physical capacity across all four sites, enabling to accommodate the surge of critically ill patients and safe transfers between the hospitals of the organisation;

- introduction of dedicated teams, allowing to streamline very complex, albeit repetitive procedures such as rapid intubations of clinically deteriorating patients or proning ventilated patients;

- standardisation of medical and nursing guidelines, allowing from human factors perspective to provide reliable and consistent clinical care, despite constant rotation of medical and nursing staff;

- simplification of medicinal governance, with special emphasis on premade infusions and prefilled syringes;

- provision of personal protection in accordance with Public Health England (PHE) guidelines;

- a widespread introduction of pastoral care for medical and nursing staff allowing for easy access to wellbeing and restorative practice teams.

The above-mentioned goals were achieved in several different, interconnected and complementary ways.

Creation of control and command centres with different, but clear responsibilities allowed for constant two-way communication. The responsiveness was increased by the continuous in-hospital presence of senior management and clinicians, taking part in twelve-hour shifts. It was especially crucial when the different districts of Birmingham surged sequentially, and fast decisions on patients and personnel transfers between critical care units of our organisations were required to ensure resilience and to balance the burden of the admissions between the hospitals. To comply with social distancing rules, and reduce potential exposure, modern technologies were widely implemented, inclusive of video conferencing and messaging platforms.

The immediate (approximately four weeks before anticipated peek of COVID-19-related admissions) closure of non-emergency operating rooms, outpatient clinics, and day-case units allowed to significantly increase the available medical and nursing workforce. The massive upskilling program consisted of the provision of more than 20 standardised educational lectures and hands-on sessions, increasing critical care medical staffing by more than 200 doctors, fully compliant with national directives $[6,7]$. Initially, the focus was on non-critical care operating rooms (OR) staff, later on expanding to many other branches of practice. Doctors from a wide range of medical and surgical specialities were upskilled, inclusive such disciplines as neurology, ophthalmology or plastic surgery.

Closure of operating theatres also allowed to move anaesthetic equipment, such as ventilators, infusion pumps, and monitors to critical care as well as spare hospital beds. The physical constraints of limited space in the critical care unit were overcome by having two beds in one cubicle. This alone required detailed planning by the estates and medical engineering to ensure a sufficient supply of medical gases and fluids. The temporary layout of the ICU, although very crowded, was fully functional. The physical dividing walls were erected to separate clean areas from COVID ICU, as fully operational non-COVID ICU was maintained throughout the pandemic.

Closure of elective activities in the hospital put a strain on emergency workflow, especially in the supra-regional surgical specialities. However, highly sub-specialised procedures requiring emergency input (such as neurosurgery, burns, cardiothoracic surgery and hyperacute solid organ transplantations) were still successfully provided within the four hospitals of the Trust. The capacity in the private hospital providers, as well as NHS hospitals routinely serving non-urgent cases, was used to ensure delivery of urgent operations throughout the surge.

Within COVID designated ICU areas, streamlining of complex medical procedures allowed to deliver high quality, standardised medical management to the patient, even when the footprint in intensive care exceeded 200\% pre-COVID capacity. The key factor was the utilisation of transferable skills of many medical professionals, not routinely involved in critical care.

For example, the insertion of central lines was provided by interventional radiologists, proning of ventilated patients by neurosurgeons, and OR staff, and bedside tracheostomies by ENT (otolaryngologists) and maxillofacial surgeons. To allow consistency, checklists were employed widely, and allied health professionals such as physiotherapists were empowered to enforce them. Not surprisingly, this led to improved compliance with pre-existing medical guidelines, such us on lung-protective ventilation. An added benefit from having a multidisciplinary team approach was the availability of instant speciality input into patient displaying a plethora of multiorgan complications related to COVID- 19 .

Medicinal governance was focused on minimising possibilities of inadvertent medical errors. This was achieved by standardisation and simplification of the prescriptions. Hospital pharmacy was involved in the preparation of premixed infusions of inotropes and sedatives to minimise workload at the bed spaces [8]. Wherever there was an alternative medication allowing for less frequent or less 
burdensome administration, the change of prescription was sought. In case of any significant changes, immediate communication via control and command channels followed, allowing for a relatively quick change of standard practice. If a deviation from previous practice was deemed necessary, the change in the electronic prescription system utilised by Trust (Birmingham Systems Prescribing Information and Communications System -PICS, Birmingham, UK) was immediately implemented. One of the examples could be the increase in the dose of anticoagulants, namely enoxaparin in COVID patients in line with emerging national and international recommendations [9].

The provision of appropriate personal protection equipment (PPE) was based on Public Health England (PHE) guidelines [10]. The emphasis was not only on the sufficient supply of correct PPE to health care workers but also on making sure that PPE is applied and disposed of in each and every case in the correct way, reducing the risk of transmission and contaminations especially during the aerosol-generating procedures and particularly at the doffing [11]. This was achieved in two separate, but interconnected ways.

Outside critical care, highly specialised assessment teams, led by senior clinicians, were available around the clock to provide a clinical review of suspected patients with COVID. If the patient was pre-qualified for admission to critical care, fully donned in PPE team of the senior anesthesiologists was deployed to secure the airways and following endotracheal intubation to transfer and establish mechanical ventilation in critical care. Such an approach allowed us to minimise the risk of contamination during initial aerosol-generating procedures [12].

Inside ICU, the donning and doffing stations were established. All stations were staffed by nominated officers, with the responsibility of advising on and enforcing the predefined sequence on donning and doffing of PPE. The officers had the authority to stop anyone not following the sequence, as well as to limit flow through the stations to minimise the possibility of cross-contamination and subsequent infection. The efficacy of such an approach was further assessed by our Trust. SARS$\mathrm{CoV} 2$ seroconversion rate between critical care workers in UHB was only $14.8 \%$; hence it was lower than in the tested overall population of health care workers in our Hospitals, in whom it has reached $24.4 \%$. It may be interpreted, albeit with caution, that health and safety approach, described in detail above, allowed to minimise occupational risk, even with the significant exposure to the viral load [4]

The special emphasis was put on staff wellbeing and restorative practice, to minimise well-recognised psychological trauma caused by the pandemic [13]. The level of stress was increased as many of staff members were working outside their normal practice, with an obvious initial poor understanding of COVID-19 pandemic, especially in view of inevitable bad outcomes. Support was provided by a previously established wellbeing group consisting of senior clinicians and restorative practice nurses. In addition, quiet rooms were created to allow for rest and mindfulness, away from clinical areas. At the end of the surge, all redeployed medics were offered feedback sessions, allowing for de- briefing and further discussion.

\section{DISCUSSION}

Implementing changes in organisation of the care, fasttracked upskilling programs for staff across the board, with collaborative work of Trust employers outside they normal practice, guided by pre-written checklists with clear escalation paths, allowed our Hospital and the Trust to fully function throughout the surge of COVID-19 patients from March until mid-May 2020.

The number of patients admitted to the ICU in QEHB has been similar to the number of patients reported in the most affected critical care units in other nations, such as in Milan (Italy) or in New York (USA) [14, 15]. In total, the intensive care so far was provided to more than 180 patients tested positive for SARS-CoV-2. The overwhelming majority required mechanical ventilation, with an average length of stay in critical care of 19 days. The survival rate of closed cases (patients who either were discharged alive or died on the unit) is around 67\% which is in line with case-mix data provided nationally by the Intensive Care National Audit \& Research Centre (ICNARC) [16] detailed results of medical management for the Trust and the region are presented further in ICNARC publications, hence not analysed in detail in this correspondence (16).

\section{CONCLUSIONS}

The achieved clinical outcomes support the efficacy of the multimodal plan implemented before the surge. A relatively low seroconversion rate between critical care staff outlines the safety of the departmental approach to personal protection. The positive feedback from doctors redeployed to critical care during the pandemic shows the importance of team model and joint decision making in medical practice.

In summary, we believe that a multidisciplinary approach, used in our organisation, allowed for the provision of safe and efficient critical care throughout the surge of critically ill patients during COVID-19 pandemic.

\section{REFERENCES}

1. Department of Health and Social Care. Coronavirus (COVID-19) action plan; 03.03.2020 (download 29.05.2020) [https://www.gov.uk/ government/publications/coronavirus-action-plan].

2. NHS England and NHS Improvement. Next steps on NHS response to COVID-19.17.03.2020 (download 29.05.2020). [https://www.england. nhs.uk/coronavirus/wp-content/uploads/sites/52/2020/03/urgentnext-steps-on-nhs-response-to-covid-19-letter-simon-stevens.pdf].

3. NHS England NHS Improvement. Clinical guide for anaesthesia service reorganisation during the coronavirus pandemic 17 March 2020 Version 1 (download 27.05.2020) [https://www.england.nhs.uk/coronavirus/ wp-content/uploads/sites/52/2020/03/Specialty-guide_Critical-careand-anaesthesia-service-reorganisation_V1.pdf].

4. Shields AM, Faustini SE. SARS-CoV-2 seroconversion in health care workers. MedRxviv (online) (version 19.05.2020) https://www.medrxiv. org/conten...

5. Rhodes A, Ferdinande P, Flaatten $\mathrm{H}$, et al. The variability of critical care bed numbers in Europe. Intensive Care Med 2012;38:647-1653 
6. ICM Anaesthesia. National guidance (download 27.05.2020) [https:// icmanaesthesiacovid-19.0rg/national-guidance].

7. NHS England NHS Improvement. Adult critical care novel coronavirus (COVID-19) staffing framework 3 April 2020 [(download 27.05.2020) https://www.england.nhs.uk/coronavirus/wp-content/uploads/ sites/52/2020/03/C0087-speciality-guide-critical-care-specialtyguide-v2-.pdf]

8. Ludwin K, Filipiak KJ, Jaguszewski M, et al. Place of prefilled syringes in COVID-19 patient based on current evidence. Am J Emerg Med. 2020 May 10.1016/j.ajem.2020.05.016 [Epub ahead of print].

9. Paranjpe I, Fuster V. Association of Treatment Dose Anticoagulation with In-Hospital Survival Among Hospitalized Patients with COVID-19. J Am Coll Cardiol. 2020 May 06. 10.1016/j.jacc.2020.05.001 [Epub ahead of print]

10. Public Health England. COVID-19 personal protective equipment (PPE) [(download 27.05.2020) https://www.gov.uk/government/ publications/wuhan-novel-coronavirus-infection-prevention-andcontrol/covid-19-personal-protective-equipment-ppe].

11. Verbeek JH, Rajamaki B. Personal protective equipment for preventing highly infectious diseases due to exposure to contaminated body fluids in healthcare staff. Cochrane Database Syst Rev. 2019;7(7): CD011621. Published 2019 Jul 1.

12. Smereka J, Ruetzler K, Szarpak L, FilipiakK, Jaguszewski M. Role of mask/ respirator protection against SARS-CoV-2. Anesthes Analges. April 13, 2020 - [ahead of print] DOI: 10.1213/ANE.0000000000004873

13. Mock J. Frontline Trauma. Scien Am. 2020 June 322(6):36-37.

14. Cecconi M. Social media personal communication Linkedin.com [(download 27.05.2020) https://www.linkedin.com/feed/update/ urn:li:activity:6671001289553416192/].

15. Richardson S, Hirsch JS, Narasimhan M, et al. Presenting Characteristics, Comorbidities, and Outcomes Among 5700 Patients Hospitalized With COVID-19 in the New York City Area. JAMA. 2020;323(20):2052-2059.

16. ICNARC report on COVID-19 in critical care 22 May 2020 [(download 27.05.2020) https://www.icnarc.org/Our-Audit/Audits/Cmp/Reports]

\section{ORCID and contributionship}

Tomasz Torliński - 0000-0003-2255-5317 A,B,C,D,E,F

Lucas Rakasz - 0000-0002-7826-4669 ${ }^{B, C, D, E, F}$

Barbara Wysota - 0000-0002-2814-0561 B,C,D,E,F

Marcin Czyż - 0000-0001-7224-4272 ${ }^{B, C, D, E, F}$

Catherine Snelson - 0000-0002-1790-0780 A,B,C,D,E,F

\section{Conflict of interest}

Authors declare no conflict of interest.

\section{CORRESPONDING AUTHOR Tomasz Torliński}

Department of Anaesthetics and Critical Care, Queen Elizabeth Hospital Birmingham

University Hospitals Birmingham NHS FT,

Mindelsohn Way, B15 2TH, Birmingham, United Kingdom

e-mail: tomasz.torlinski1@nhs.net

Received: 30.05 .2020

Accepted: 03.07 .2020

A - Work concept and design, B - Data collection and analysis, C - Responsibility for statistical analysis,

D-Writing the article, $\mathbf{E}$-Critical review, $\mathbf{F}$ - Final approval of the article 